\title{
Neural correlates of habituation and dark adaptation in the visual cortex of the rat*
}

\author{
RAYMOND T. BARTUS $\dagger$ and STEPHEN H. FERRIS $\dagger \dagger$ \\ Vision Branch, Naval Submarine Medical Research Lab, Groton, Connecticut 06340
}

\begin{abstract}
Sixteen rats were chronically implanted with monopolar electrodes over the primary visual cortex. Averaged evoked responses to repetitive flashes of light were evaluated during habituation and dark adaptation at two stimulus intensity levels. It was found that no consistent changes occurred in the early evoked response components if the rats were dark adapted prior to testing. However, significant increases in amplitude of the early components did occur over trials in nondark-adapted rats, with greatest increases occurring with the dimmer of the two stimulus intensities. These data were interpreted as demonstrating that the early evoked response components constitute a neurophysiological analog to psychophysical functions of brightness perception. Contrary to these effects, the later evoked response components were not influenced by dark adaptation. However, significant changes did occur over repeated stimulus presentations which are most easily accounted for by concepts of habituation. Increases as well as decreases in amplitude were observed depending upon the particular component, with the largest and more prolonged effects resulting from stimulation with the less intense light. These characteristic changes in averaged evoked responses support the notion that early and late components represent two different classes of neural events, and support a "stimulus-model" theory of habituation, but are inconsistent with an "afferent-inhibition" theory.
\end{abstract}

Habituation is defined most simply as a decrease in behavioral responsiveness to repeated stimulation, which results from factors other than receptor adaptation or fatigue. The implications that central neural mechanisms are responsible for this change in responsiveness and that basic mechanisms of sensory processing and behavioral plasticity may be involved have prompted considerable research and theorizing.

One popular approach adopted in an effort to define the underlying mechanisms responsible for habituation has been to record electroencephalograms (EEGs) and evoked responses (ERs) during repeated sensory stimulation trials to identify the changes in neural activity that occur. A cursory examination of this literature suggests that ERs recorded in the specific sensory pathways exhibit significant decrements in amplitude over the course of habituation (e.g., Regan, 1972; Perry \& Childers, 1969). However, a closer look at the literature reveals a discouraging amount of contradictory evidence and inconsistent conclusions. Although several authors have indeed observed decreases in the amplitude of the sensory evoked response, much of that data has been criticized for lack of adequate experimental control. For example, Guzman-Flores et al

*This research was conducted while the first author held a National Research Council postdoctoral associateship and was partially supported by Research Work Unit MF5.524.004-9015DA5G from the Bureau of Medicine and Surgery, U.S. Navy Department. Thanks are extended to John Marriott and Jo Ann S. Kinney for helpful comments on the manuscript and to Alma Ryan for assistance in the statistical analyses.

+Now at Research Division, Parke-Davis and Company, Ann Arbor, Michigan 48106.

+Now at New York University Medical Center, New York, New York 10003
(1960) and Fernandez-Guardiola et al (1961) demonstrated that decreases in amplitude may sometimes result from peripheral influences, such as inner ear muscle contraction or pupillary constriction, while Marsh, Worden, and Hicks (1962) found that significant changes in ER amplitude occur with rather subtle changes in the Ss' body position. Also, Worden and Marsh (1963) demonstrated that unless stringent statistical procedures are used when evaluating changes in ER amplitude, incorrect conclusions may be drawn about chance effects. Thus, the attenuation that all of these authors observed was not due to actual changes in neural responsiveness to the stimulation, but rather to other less interesting, confounding variables. The possibility that still other undefined variables have also influenced changes in ERs reported by others in the past is quite apparent, for different authors have reported either no measurable decreases, inconsistent changes, or even substantial increases in ER amplitude with repeated stimulation (for critical reviews, see Thompson, 1967; Thompson \& Spencer, 1966).

For this reason, the present research addressed itself to a reexamination of changes in the sensory ERs accompanying repeated stimulation to determine whether such changes can be attributed to habituation or, if not, whether possible confounding sources can be identified.

\section{METHOD}

\section{Subjects and Surgery}

Sixteen male albino rats (Charles River CD strain) weighing approximately $600 \mathrm{~g}$ were used as Ss. Details of the chronic electrode implantation technique have been described previously 


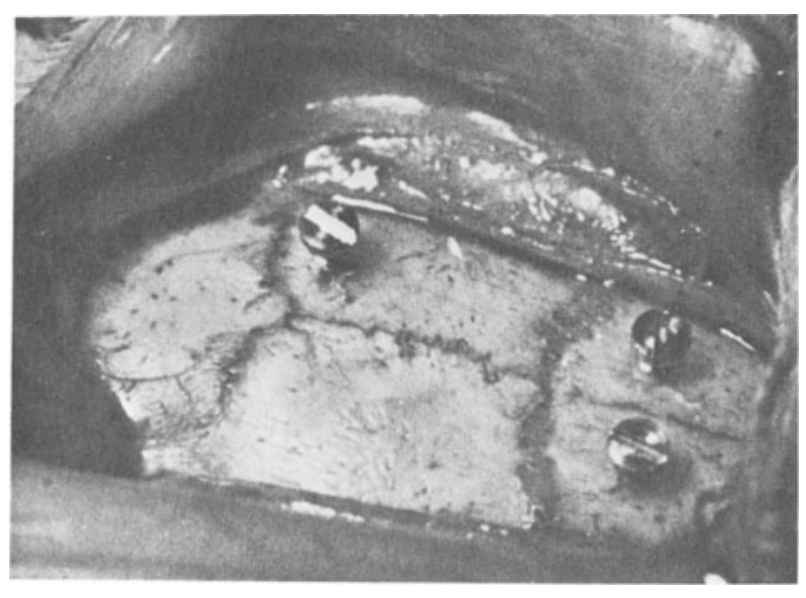

Fig. 1. Photograph depicting relative positions of cortical, reference, and ground electrodes, attached to the rat's skull.

(Ferris \& Bartus, 1973). Briefly, miniature self-tapping stainless steel screws (size $00,1 / 8$ in. long) placed over the rat's primary visual cortex ( $3 \mathrm{~mm}$ lateral and $2 \mathrm{~mm}$ anterior to the lambda) were used as monopolar epidural electrodes. Reference and ground electrodes, consisting of similar screws, were positioned near the ipsilateral and contralateral frontal sinuses, respectively ( $3 \mathrm{~mm}$ lateral and $5 \mathrm{~mm}$ anterior to the bregma, see Fig. 1). These screw electrodes were connected to a miniature Amphenol stripconnector socket using Teflon-coated stainless steel wire. The screws and socket assembly were then embedded on the skull in a mound of dental acrylic. Following surgery, a recovery period of at least 2 weeks was given to each rat before experimental testing was initiated.

\begin{abstract}
Apparatus
Testing was conducted in a totally dark room, shielded from 60-cycle noise and high-frequency interference. Gross body movements were restricted by placing the unanesthetized rat in a clear Plexiglas restraining device (see Ferris \& Bartus, 1973). An opaque rectangular box measuring $27.94 \times 43.18 \times 27.94 \mathrm{~cm}$ high and painted with a semigloss silver interior was placed over the restrainer. The stimuli used for eliciting the visual evoked responses (VERs) were discrete flashes of light produced by the light source from a Grass PS-2 photo stimulator. This light source was positioned over an opening in the top of the opaque box, directly above the rat's head. The rat's EEG was amplified by a Grass P511 preamplifier, and the VERs were obtained using a computer of average transients. Each VER was computed on-line by summating the ongoing EEG that was time-locked to 50 individual flashes of light, and permanent records of the VERs were immediately printed using an $\mathrm{x}, \mathrm{y}$ plotter.
\end{abstract}

\section{Procedure}

The stimulus flashes were projected from the photo stimulator at a rate of $1 / \mathrm{sec}$, with two different intensity levels used. The brighter intensity was produced by setting the Grass PS- 2 photo stimulator intensity on Level No. 8, while the dimmer stimulus was produced by adjusting the photo stimulator intensity to Setting No 1 and using the 1.0 neutral density filter to further reduce the intensity by $90 \%$. These stimulus parameters thus provided two intensity levels which differed by nearly two log units.

On the basis of preliminary indications resulting from pilot work, the influence of dark adaptation level was also evaluated for its effects on successive VER changes and possible interaction with VER habituation. Two different levels of dark adaptation were used. In the "no dark adaptation" condition, the rats were placed in the darkened experimental room about 3 min prior to testing. In the "dark adaptation" condition, an interval of 30-35 min of to tal darkness elapsed before testing was initiated. Four rats were assigned to each of the four treatment combinations, so that each rat was tested at only one intensity level and only one state of dark adaptation. The experimental session consisted of obtaining 10 successive VERs, with a 90-sec intertrial interval occurring between each set of 50 flashes.

For the data analysis, the amplitudes of the main components (see Fig. 2) were measured, with the amplitude defined as the peak to peak deflection from the previous component. The data from the 1050 -sec trials were blocked in to five successive pairs. Using the amplitudes for Trial Block 1 as the baseline, the amplitudes of the four remaining blocks were expressed as a percent change from that baseline.

\section{RESULTS}

The major findings of this investigation can be summarized by stating that the earlier components of the VER were highly affected by changes in level of dark adaptation but were apparently not influenced by habituation, while the later VER components proved most sensitive to habituation but were not affected by the level of dark adaptation. However, in order to eliminate the criticism that chance variation in VER amplitude was not taken into account (Thompson, 1967; Thompson \& Spencer, 1966; Worden \& Marsh, 1963), the data in the present study were subjected to stringent statistical, evaluation. These data were first analyzed using a four-way analysis of variance (stimulus intensity by dark adaptation level by trial block by VER component), with repeated measures over the last two factors. This analysis revealed significant three-way interactions involving intensity (by component by trial block: $F=1.65, \mathrm{df}=18 / 216, \mathrm{p}<.05)$ and dark adaptation (by component by trial block: $\mathrm{F}=1.81$, $\mathrm{df}=$ $18 / 216, p<.05)$. Separate three-way analyses were then carried out for each VER component before the main effects were evaluated.

The significant interactions $(p<.05)$ revealed by these analyses are evident in Figs. 3 and 4. As shown in Fig. 3, the amplitudes of the first three components did

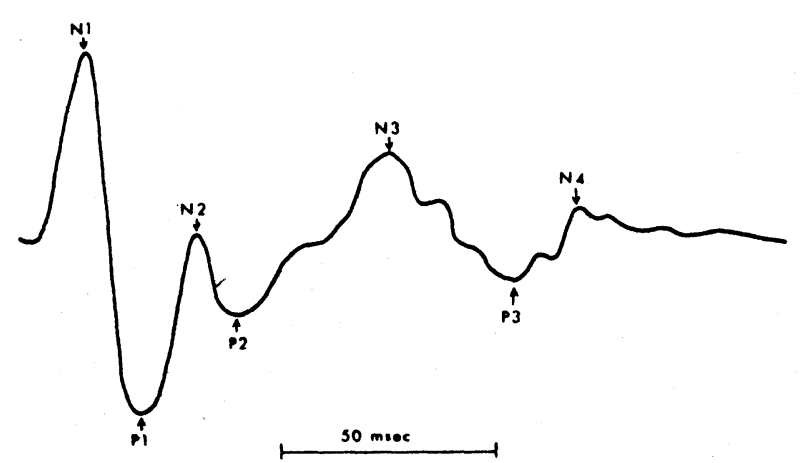

Fig. 2. Schematic representation of typical VER obtained from 50 sweeps of signal averager. The most consistent and prominent components are descriptively labeled with respect to polarity and relative position. 
not change over successive trial blocks when the animals were dark adapted. Without dark adaptation, however, these components gradually increased in amplitude during the test session, continuing to show a significant effect $25 \mathrm{~min}$ after testing was initiated. It is evident from Fig. 3 that the largest amplitude increases in nondark-adapted Ss occurred in the earliest component (N1), with progressively less of an increase occurring in $\mathrm{P} 1$ and $\mathrm{N} 2(\mathrm{~N} 1-\mathrm{F}=7.57 ; \mathrm{P} 1-\mathrm{F}=13.91 ; \mathrm{N} 2-\mathrm{F}=9.94$; df $=1 / 12$ ). Finally, although not shown in Fig. 3, these analyses indicated that the increases due to dark adaptation were greater for the low-intensity than for the high-intensity flash $(\mathrm{N} 1-\mathrm{F}=4.97 ; \mathrm{P} 1-\mathrm{F}=8.27$; $\mathrm{N} 2-\mathrm{F}=9.49 ; \mathrm{df}=1 / 12$ ).

In contrast to these changes in the early components, the later VER components were not influenced by level of dark adaptation ( $p>.05)$. Nevertheless, significant changes did occur (Fig. 4). The amplitude of P2
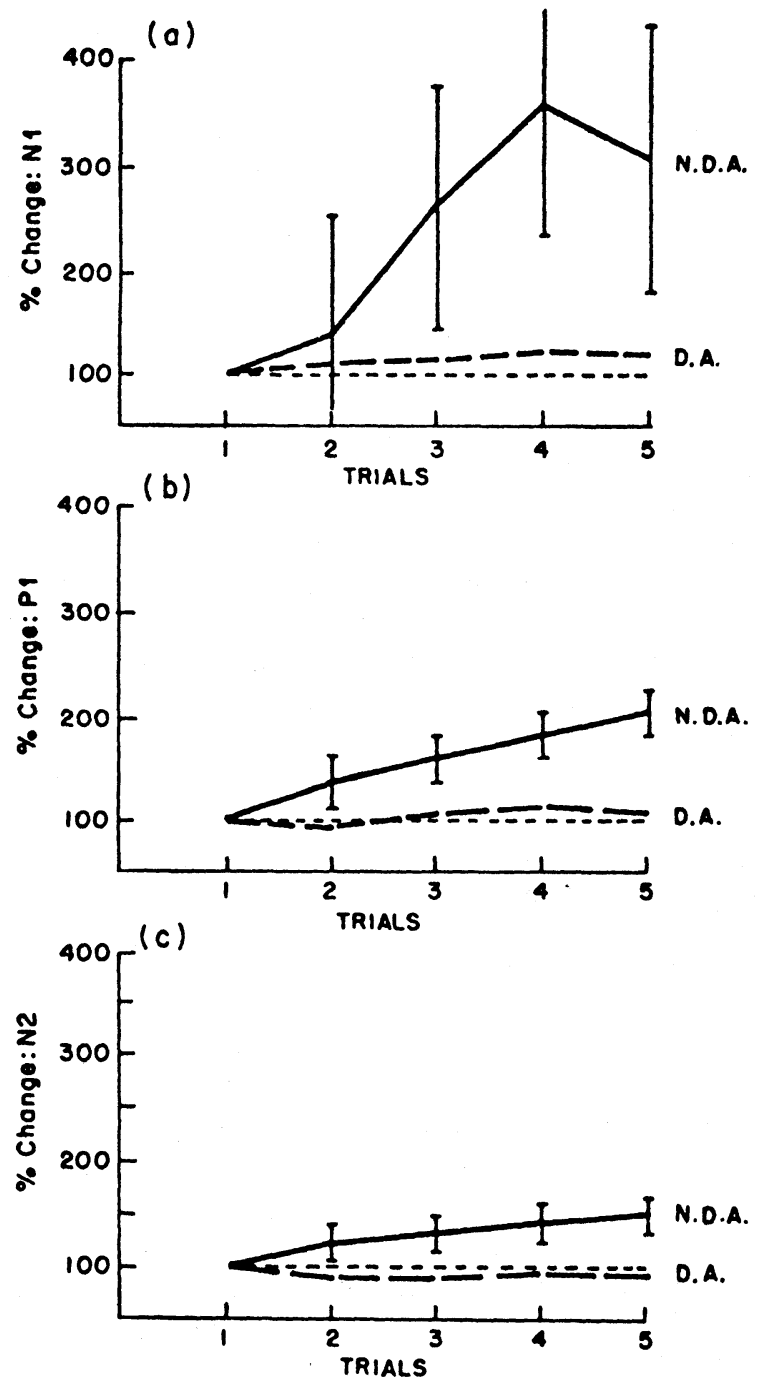

Fig. 3. Changes in early VER components occurring over successive trial blocks in dark-adapted (D.A.) and nondark-adapted (N.D.A.) rats. Vertical bars represent the .05 critical difference scores computed from each respective analysis of variance.
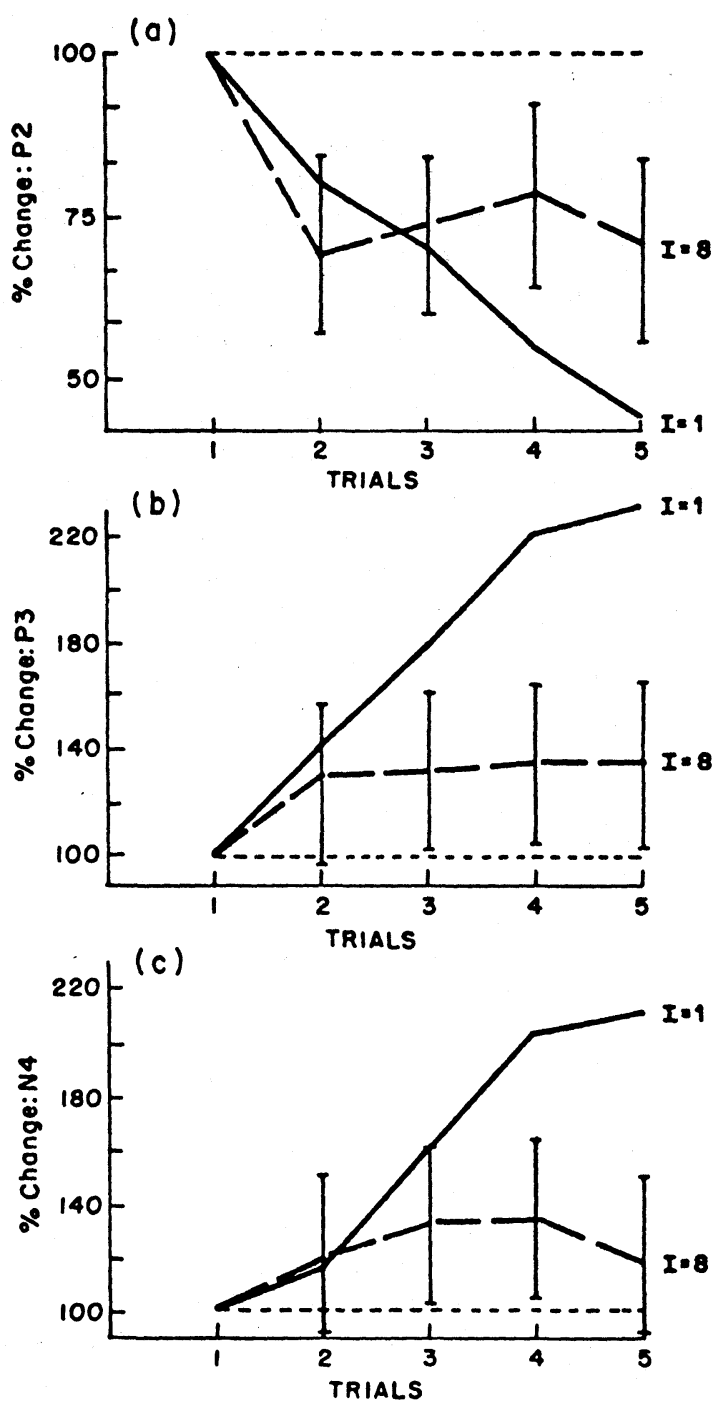

Fig. 4. Changes in later VER components occurring over successive trial blocks in the high stimulus intensity groups (I = 8 ) and low stimulus intensity groups $(I=1)$. Vertical bars again represent the .05 critical difference scores computed from each respective analy sis of variance.

decreased over successive trial blocks $(\mathrm{F}=4.44$, $\mathrm{df}=$ $3 / 36)$ and the amplitudes of $\mathrm{P} 3$ and $\mathrm{N} 4$ increased (F = $6.75 ; \mathrm{df}=7.14 ; \mathrm{df}=3 / 36)$, while the amplitude of $\mathrm{N} 3$ $(F=2.41$, df $=3 / 36$; not shown) did not change. Furthermore, the magnitude of the changes which occurred in these later components was highly dependent upon the flash intensity. At the low intensity, the changes were large and continued to increase with each successive trial block. At the high intensity, however, the changes were relatively small, and little, if any, further change occurred after Trial Block 2. All of these differences were significant at the .05 probability level, or better.

\section{DISCUSSION}

The results of this study clearly demonstrate that 
reliable changes do occur in the rat's cortical VER during the course of repeated stimulation. However, not all of the changes that occurred can be attributed to the effects of habituation. For example, changes in the early components did not occur if the rats were sufficiently dark adapted before testing, and it is suggested that the changes that occurred in these particular components simply reflect an increase in subjective stimulus intensity. In other words, as the amount of unbleached rhodopsin increased during testing of the nondark-adapted rats, the apparent brightness of the flashing light presumably increased, with the amplitude of the early VER components also increasing in these Ss. Thus, according to this interpretation, the significant changes in amplitude in the early components represent a neurophysiological analog to psychophysical functions of stimulus intensity.

These results also demonstrate that changes in the level of dark adaptation, which were still evident after $25 \mathrm{~min}$ of total darkness, constitute another confounding variable which must be controlled when changes in the amplitude of the VER are being studied. Recent evaluations of cortical and subcortical VERs from dark-adapted and nondark-adapted cats indicate that this phenomenon is not peculiar to VERs obtained from the cortex of the albino rat (Bartus, unpublished observations, 1973).

Unlike the changes in the early components of the rat's VER, the later components were not influenced by the degree of dark adaptation. Nevertheless, significant changes with repeated trials did occur, and an obvious question concerned the extent to which these changes represented true habituation effects. Two other possibilities have been proposed and have provided a major source of criticism of past studies (see Thompson, 1967; Thompson \& Spencer, 1966). One suggestion is that the effects are due to peripheral influences such as pupillary contraction and changes in receptor orientation due to head movement, which thus alter the effective stimulus intensity. The other suggestion is that the effects are the result of simple receptor fatigue. The experimental procedure described above was designed to minimize both of these possibilities, and close examination of the data suggested that these effects were, in fact, negligible.

For example, by using the albino rat, whose unpigmented iris has little light absorption capabilities, and an experimental arrangement which had proven to provide consistent VERs from trial to trial (see Ferris \& Bartus, 1973), it was hoped that influences from peripheral changes would be minimal. Since no changes occurred in the early VER components (providing the rats were sufficiently dark adapted) and since the changes that did occur in the late components were manifested as increases as well as decreases (depending upon the particular component), any influence due to consistent peripheral changes can be effectively ruled out.
On the other hand, it was hoped that receptor fatigue could be eliminated by arranging the experimental procedure so that the intertrial interval was of long enough duration to enable the retina to recover adequately from the repeated stimulation of each individual trial. Again, the data indicate that this attempt was successful. For example, no decreases occurred in the early components, as would be predicted on the basis of receptor fatigue. The decrease that did occur in the later component was not only smaller for the more intense stimulus, but reached asymptote sooner as well. These findings are, of course, the opposite of what would be expected if the visual system fatigued, but are perfectly consistent with predictions based on studies of behavioral habituation (Groves \& Thompson, 1970; Thompson \& Spencer, 1966). For these reasons, it was concluded that the changes that occurred in the late components of the VER to repeated stimulation are accounted for most easily by the concept of habituation. This interpretation also agrees with the reports of other authors who found that habituation of the auditory evoked response over prolonged sessions was restricted to the later components (Hall, 1968; Wickelgren, 1968).

The finding that the effect of habituation was evident only in the later components, while the effect of dark adaptation was limited to the early components, is consistent with the notion that the later portion of the VER is most often correlated with psychological variables, while the earlier portion of the VER is primarily correlated with the physical characteristics of the sensory input (Ruchin \& John, 1966). If this distinction between early and later components is accurate, as the present data also suggest, then Hernandez-Peón's (1960) "afferent neuronal inhibition" theory of habituation may be severely compromised. This theory contends that habituation results from an inhibition of sensory input at several or all synaptic levels along the sensory pathway. Such a conceptualization would seem to be inconsistent with the present data, for the effects of habituation are not apparent in the earlier components of the cortical VER, which are presumably correlated with sensory input.

Conversely, the significant changes that did occur in the later components are consistent with other theories of habituation which argue for some change in the output side of the system. For example, Sokolov's (1963) "stimulus-model" theory of habituation does not assume that the incoming sensory stimulus is modified during habituation, but rather that it is compared to a model that was formed in the sensory cortex during previous occurrences of the same or similar sensory events. If the incoming stimulus is novel, it will not match the model stimulus and an orienting reflex is elicited by the reticular formation. If the incoming sensory event occurred before and the two neural representations do match, then orienting is attenuated and the overt response to the stimulus is reduced. 
This theory of habituation has striking similarities to John's (1967) neural theory of memory. John assumes that the performance of a conditioned response is, in part, dependent upon a comparison of the neural representation of the incoming sensory information and the neural representation of whatever is stored in that part of memory elicited by the sensory input. He argues that the later components of the VERs accompanying learning actually represent activity related to the neural readout of information from memory (John, 1967, 1972). He and others have shown that although no changes occur in the early VER components, the later components undergo significant changes as learning progresses (John, Ruchin, \& Villegas, 1963; Begleiter \& Platz, 1969; Spinelli \& Pribram, 1972; Pribram, Spinelli, \& Kamback, 1967). Furthermore, the changes that occurred during learning are absent on those trials when the conditioned response (CR) fails to occur (John \& Morgades, 1969) and disappear when behavioral extinction occurs (Begleiter \& Platz, 1969). It has also been shown that a novel stimulus will elicit those changes that occurred during learning when behavioral generalization (i.e., the CR) occurs to the novel stimulus (Ruchin \& John, 1966). Finally, the behavioral choice of a trained animal, whether correct or incorrect, can be reliably predicted in a two-choice discrimination task, on the basis of the shape of the preceding late components which are elicited by the discriminative stimulus (John, Shimokochi, \& Bartlett, 1969; John, Bartlett, \& Shimokochi, 1969). Of course, none of these phenomena occur in the untrained animal, even when the $\mathrm{CR}$ is made by chance.

If John's notion is accurate (i.e., that these later components represent readout from memory), then one could predict on the basis of Sokolov's model of habituation that these components should show progressive changes with habituation, while the earlier components should not. In other words, as the stimulus is repeatedly presented and a neural model is presumably being formed in memory, changes in the later components of the VER ought to occur which reflect these events. Since, according to Sokolov, it is the stimulus model that is altered and not the afferent stimulus input, no such changes would be expected to occur in the early components. Of course, the data of the present study are perfectly consistent with these predictions and are therefore offered as support for both Sokolov's model of habituation and John's interpretation of changes occurring in the VER during learning. Although the conclusions drawn here are not without contest, it is suggested that they may nevertheless provide an additional data base for using VERs to study neural mechanisms of habituation, as well as for using VER habituation to study neural mechanisms of memory.

\section{REFERENCES}

Begleiter, H., \& Platz, A. Evoked potentials: Modifications by classical conditioning. Science, 1969, 166, 769-771.

Fernandez-Guardiola, A., Roldan, E., Fanjul, L., \& Castells, C. Role of the pupillary mechanism in the process of habituation of the visual pathways. Electroencephalography \& Clinical Neurophy siology, $1961,13,564-576$.

Ferris, S. H., \& Bartus, R. T. A method for recording and analyzing EEG's and VER's from rats under hyperbaric conditions. Naval Submarine Medical Research Lab Report No. 747, 1973, Groton, Connecticut.

Groves, P. M., \& Thompson, R. F. Habituation: A dual-process theory. Psychological R eview, 1970, 77, 419-450.

Guzman-Flores, C., Alcaraz, M., \& Harmony, T. Role of the intrinsic ear muscles in the process of acoustic habituation. Boletin del Instituto por la Estudia de Medica Biologica, 1960, $18,135-140$

Hall, R. D. Habituation of evoked potentials in the rat under conditions of behavioral control. Electroencephalography \& Clinical Neurophysiology, 1968, 24, 155-165.

Hernandez-Peón, R. Neurophysiological correlates of habituation and other manifestations of plastic inhibition (internal inhibition). Electroencephalography \& Clinical Neurophysiology, 1960, Suppl. 13, 101-114.

John, E. R. Mechanism s of memory. New York: Academic Press, 1967.

John, E. R. Switchboard versus statistical theories of learning and memory. Science, 1972, 177, 850-864.

John, E. R., Bartlett, F., \& Shimokochi, M. Neural readout from memory during generalization. $16-\mathrm{mm}$ sound and color film. New York: Brain Research Laboratories, New York Medical College, 1969.

John, E. R., Ruchkin, D. S., \& Villegas, J. Signal analysis of evoked potentials recorded from cats during conditioning. Science, $1963,141,429-431$.

John, E. R., \& Morgades, P. P. Neural correlates of conditioned responses studied with multiple chronically implanted moving microelectrodes. Experimental Neurology, 1969, 23, 412-425.

John, E. R., Shimokochi, M., \& Bartlett, F. Neural readout from memory during generalization. Science, 1969, 164, 1519-1521.

Marsh, J. T., Worden, F. G., \& Hicks, L. Some effects of room acoustics on evoked auditory potentials. Science, 1962, 137, 281-282.

Perry, N. W., \& Childers, D. G. The human visual evoked response. Springfield, Ill: Thomas, 1969.

Pribram, K. H., Spinelli, D. N., \& Kamback, M. C. Electrocortical correlates of stimulus, response, and reinforcement. Science, 1967, 157, 94-95.

Regan, D. Evoked potentials in psychology, sensory physiology and clinical medicine. London: Chapman \& Hall, 1972.

Ruchkin, D. S., \& John, E. R. Evoked potential correlates of generalization. Science, 1966, 153, 209-211.

Sokolov, Y. N. Perception and the conditioned reflex. (Trans. S. W. Waydenfeld) Oxford: Pergamon Press, 1963.

Spinelli, D. N., \& Pribram, K. H. Neural correlates of stimulus response and reinforcement. Brain Research, 1970, 17, 377-385.

Thompson, R. F. Foundations of physiological psychology. New York: Harper \& Row, 1967.

Thompson, R. F., \& Spencer, W. A. Habituation: A model phenomenon for the study of neuronal substrates of behavior. Psychological Review, 1966, 173, 16-43.

Wickelgren, W. O. Effect of acoustic habituation on click-evolved responses in cats. Journal of Neurophysiology, 1968, 31, 777-784.

Worden, F. G., \& Marsh, J. T. Amplitude changes of auditory potentials evoked at cochlear nucleus during acoustic habituation. Electroencephalography \& Clinical Neurophy siology, 1963, 15, 866-881.

(Received for publication November 15, 1973; accepted November 29, 1973.) 